\title{
Aspectos morfofisiológicos das cultivares de cafeeiro Catuaí-Vermelho e Conilon(1)
}

\author{
Luciana Marques de Carvalho(2), Eldo Antônio Monteiro da Silva( ${ }^{(3)}$, Aristéa Alves Azevedo ${ }^{(3)}$, \\ Paulo Roberto Mosquim ${ }^{(3)}$ e Paulo Roberto Cecon ${ }^{(4)}$
}

\begin{abstract}
Resumo - O objetivo deste trabalho foi comparar cafeeiros das espécies Coffea arabica L. cv. CatuaíVermelho e C. canephora Pierre cv. Conilon quanto à fotossíntese líquida e a aspectos da anatomia foliar a esta relacionados. Verificaram-se taxas fotossintética líquida, transpiratória e de condutância estomática maiores em plantas de C. canephora. As plantas de C. arabica apresentaram menor eficiência do sistema "antena", e folhas menores, porém mais espessas. As duas espécies diferiram, ainda, quanto ao tipo, dimensões e número dos estômatos presentes na epiderme foliar: paracíticos em C. arabica e actinocíticos em C. canephora, e maiores, mas em menor número, em C. arabica.
\end{abstract}

Termos para indexação: Coffea arabica, Coffea canephora, fotossíntese, estômato, anatomia vegetal, reações bioquímicas.

\section{Morphophysiological aspects of Catuaí-Vermelho and Conilon coffee cultivars}

\begin{abstract}
The objective of this work was to compare plants of the Coffea arabica L. cv. CatuaíVermelho and C. canephora Pierre cv. Conilon species in relation to the net photosynthesis rate and aspects of leaf anatomy. Coffea canephora plants presented higher net photosynthetic rate, transpiratory rate and leaf conductance rate than C. arabica plants, which presented smaller photochemical capacity of photosystem I, and shorter and thicker leaves than C. canephora. Both species differed also in relationship to the type, number and dimensions of stomata in the leaf epiderm: they are paracytics in C. arabica and actinocytics in C. canephora, and they are larger, but in smaller number in C. arabica.

Index terms: Coffea arabica, Coffea canephora, photosynthesis, stomata, plant anatomy, biochemical reactions.
\end{abstract}

\section{Introdução}

O gênero Coffea apresenta aproximadamente 100 espécies de cafeeiros, mas apenas cinco são cultivadas comercialmente, e entre estas, Coffea arabica L. (cafeeiro arábica) e C. canephora Pierre (cafeeiro robusta) são as mais comercializadas (Fazuoli, 1986). No Brasil, Coffea arabica constitui a maior parte dos cafeeiros plantados (Borin, 1971; Fazuoli, 1986), no

\footnotetext{
(1) Aceito para publicação em 3 de março de 2000.

(2) Av. Brasília 204, ap. 101, Bairro Fátima, CEP 36570-000 Viçosa, MG. E-mail: lumarques@buynet.com.br

(3)Universidade Federal de Viçosa (UFV), Dep. de Biologia Vegetal, Campus Universitário, CEP 36571-000 Viçosa, MG. E-mail: esilva@mail.ufv.br, aazevedo@mail.ufv.br, mosquim@mail.ufv.br

(4)UFV, Dep. de Informática. E-mail: cecon@dti.ufv.br
}

entanto, a área de plantio do robusta vem sendo ampliada, principalmente no Estado do Espírito Santo, Mato Grosso e Rondônia. Isso se deve principalmente à menor sensibilidade de $C$. canephora Pierre ao fungo Hymeleia vastatrix. Apresenta ainda vantagens comerciais, como preço reduzido no mercado internacional e utilização na indústria de café solúvel e em misturas com o arábica, o que diminui o custo do produto final (Fazuoli, 1986).

Dedecca (1957) descreveu a anatomia e o desenvolvimento ontogenético de Coffea arabica L. var. typica Cramer. Posteriormente, poucos trabalhos em anatomia têm sido desenvolvidos com esta espécie, destacando-se o de Voltan et al. (1992), e menos ainda com Coffea canephora Pierre. Estudos morfofisiológicos são poucos na literatura, mas alguns estudos comparativos foram feitos entre espécies e cultivares de cafeeiro (Nunes et al., 1969; 
Sondahl \& Crocomo, 1974; Sondahl et al., 1976; Da Matta, 1995).

O objetivo deste trabalho foi comparar as cultivares Coffea canephora Pierre cv. Conilon e C. arabica L. cv. Catuaí-Vermelho quanto ao processo fotossintético e aspectos da anatomia foliar.

\section{Material e Métodos}

O trabalho foi conduzido no Departamento de Biologia Vegetal da Universidade Federal de Viçosa, MG As mudas de Coffea canephora Pierre cv. Conilon (linhagem 81.11, clone 29), obtidas por propagação vegetativa a partir do ramo ortotrópico da planta-matriz, foram fornecidas pela Empresa Capixaba de Pesquisa Agropecuária (Marilândia, ES), e as mudas de C. arabica L. cv. Catuaí-Vermelho (UFV 2144, linhagem 2077-2-5-44), obtidas a partir de sementes, foram fornecidas pela Empresa Agropecuária do Estado de Minas Gerais (Viçosa, MG).

Cada muda, com cerca de quatro meses, foi cultivada em vaso com aproximadamente cinco litros de capacidade, contendo uma mistura de solo (Latossolo VermelhoAmarelo distrófico) e esterco de curral curado, na proporção de $70 \%$ e $30 \%$, respectivamente. Os cafeeiros foram cultivados em casa de vegetação, de outubro de 1995 a março de 1996, sendo irrigados diariamente, ou sempre que necessário. Uma semana após o transplante, as plantas receberam adubação foliar com a solução de Clark completa.

Foram determinadas, nas plantas de ambas as espécies, as taxas de fotossíntese líquida, de transpiração e de condutância estomática, a temperatura foliar interna e a concentração de $\mathrm{CO}_{2}$ na cavidade subestomática, por meio do analisador portátil de gases a infravermelho, modelo LCA-2 (Analytical Development Co. Ltd., Hoddeston, Inglaterra). Essas medições foram feitas em sala com temperatura ambiente mantida por meio de condicionador de ar, em torno de $20 \pm 1^{\circ} \mathrm{C}$, concentração atmosférica de $\mathrm{CO}_{2}$ de $300 \mu \mathrm{mol} \mathrm{mol}^{-1}$, e taxa de fluxo de fótons saturante de 900 e $1.100 \mu \mathrm{mol}$ fótons $\mathrm{m}^{-2} \mathrm{~s}^{-1}$, previamente determinada mediante curva de irradiância de saturação, nas plantas das cultivares Conilon e Catuaí-Vermelho, respectivamente. O fluxo de fótons foi fornecido por lâmpadas de halogênio, situadas acima das plantas e separadas destas por uma camada de cerca de $10 \mathrm{~cm}$ de água. A distância das plantas em relação à fonte de luz determinava a quantidade de luz que chegava à planta. A emissão de fluorescência inicial, máxima e variável, e a estimativa da capacidade fotoquímica do fotossistema II (dado pela razão entre fluorescência variável e fluorescência máxima) foram determinadas por um fluorômetro portátil "Plant Efficiency Analyser", PEA, da Hansatech Instruments Ltda, modelo P0 2.002 (King Lynn, Norfolk, Inglaterra). Estas medições foram feitas após pré-condicionamento no escuro, por uma hora, para garantir o estado oxidado dos centros de reação fotossintéticos. Todas as medições foram feitas pela manhã, nas folhas apicais abertas, com comprimento foliar variando entre 140 e $170 \mathrm{~mm}$.

No estudo anatômico, tiras foliares com até $5 \mathrm{~mm}$ de largura, próximas e paralelas às margens, foram obtidas a partir das folhas abertas do segundo par apical. As amostras foliares foram fixadas em glutaraldeído $3 \%$ a $4^{\circ} \mathrm{C}$, por 30 horas; em seguida, lavadas em tampão fosfato de potássio $0,1 \mathrm{M}, \mathrm{pH} 7,5$, desidratadas em série alcoólica progressiva e incluídas em historresina (Technovit, da Khulzer). Para estudo em microscopia de luz, cortes de $5 \mu \mathrm{m}$ de espessura foram feitos a partir do material emblocado, com micrótomo rotativo (Jung), coloridos com azul-detoluidina, montados em bálsamo-do-canadá entre lâmina e lamínula (Berlyn \& Miksche, 1976). A observação e documentação foram realizadas em fotomicroscópio ZeissDucoval. A espessura da lâmina foliar, do mesófilo, do parênquima paliçádico e do parênquima lacunoso foi estimada medindo-se esses parâmetros em dez campos microscópicos de cada material em estudo, com o auxílio de ocular de tambor micrométrico. A espessura das epidermes abaxial e adaxial foi obtida por diferença. Foram realizados ainda cortes paradérmicos, a mão livre, nas lâminas foliares. Estes foram montados entre lâmina e lamínula e observados em microscópio óptico

$\mathrm{O}$ experimento foi realizado no delineamento inteiramente casualizado, com cinco repetições. A unidade experimental foi constituída de um vaso com uma planta. Os dados foram submetidos a análise de variância, e as diferenças entre tratamentos foram testadas pelo teste de Tukey a 5\% de probabilidade

\section{Resultados e Discussão}

As taxas de fotossíntese líquida, de transpiração, e de condutância estomática determinadas nos cafeeiros da espécie Coffea canephora cv. Conilon foram significativamente maiores do que nos cafeeiros C. arabica cv. Catuaí-Vermelho (Tabela 1). Quanto à temperatura foliar interna, no entanto, as duas cultivares não diferiram estatisticamente.

Segundo Nunes et al. (1969) e Sondahl et al. (1976), os valores das taxas de fixação de dióxido de carbono $\left(\mathrm{CO}_{2}\right)$ de plantas de $C$. canephora são, em geral, inferiores aos de Coffea arabica, apesar de 
haver alguma variação genética entre as diversas cultivares estudadas. Os baixos valores da taxa fotossintética líquida de cafeeiros podem, segundo Tió (1962), ser explicados pelo aumento da temperatura foliar durante as medições, o que pode ocorrer graças à iluminação direta. Apesar de, neste trabalho, a temperatura ambiente ter sido mantida em torno de $20 \pm 1^{\circ} \mathrm{C}$, a temperatura foliar variou de $24^{\circ} \mathrm{C}$ a $31{ }^{\circ} \mathrm{C}$, em virtude do tipo da fonte de luz utilizada e da distância da planta em relação a esta.

Os valores de taxa fotossintética líquida observados nos cafeeiros da espécie Coffea canephora foram similares aos obtidos por outros autores, como Sondahl et al.(1976) e Da Matta (1995). Sondahl et al. (1976) obtiveram, em cafeeiros da espécie C. canephora Pierre cv. Guarini, taxas fotossintéticas líquidas em torno de $3,96 \mu \mathrm{mol} \mathrm{m}^{-2} \mathrm{~s}^{-1}$ de $\mathrm{CO}_{2}$, enquanto Da Matta (1995) obteve taxas de 2,8 e $4,9 \mu \mathrm{mol} \mathrm{m}{ }^{-2} \mathrm{~s}^{-1}$ de $\mathrm{CO}_{2}$ em cafeeiros Conilon cultivados, sob condições similares, no inverno e no verão, respectivamente. Os valores determinados nas plantas da espécie Coffea arabica cv. Catuaí-Vermelho foram similares aos valores obtidos por Nutman (1937) e Da Matta (1995), os quais também determinaram a taxa fotossintética em folhas inteiras. Entretanto, esses valores são relativamente baixos, se comparados aos descritos por Tió (1962), Nunes et al. (1968, 1969), Bierhuizen et al. (1969), Sondahl et al. (1976), Yamaguchi \& Friend (1979), Kumar \& Tieszen (1980), Frischknecht et al. (1982) e Carelli et al. (1999), que em sua maior parte utilizaram a planta inteira ou discos foliares. As estimativas das taxas fotossintéticas na maioria desses trabalhos foram feitas, como neste estudo, mediante o uso do analisador de $\mathrm{CO}_{2}$ ao infravermelho, mas a cultivar estudada ou o método adotado variaram. Além disso, verificou-se, neste experimento, aumento considerável na temperatura foliar interna durante as me- dições fotossintéticas, o que, segundo Nunes et al. (1968), pode contribuir para certa redução nos valores das taxas de condutância estomática e de fotossíntese líquida.

Os cafeeiros da cultivar Catuaí-Vermelho apresentaram maiores médias de emissão de fluorescência do que os cafeeiros Conilon (Tabela 2), o que sugere que as folhas do cafeeiro $C$. canephora $\mathrm{cv}$. Conilon apresentam maior eficiência do "sistema antena" para a absorção e transferência de energia luminosa do que os cafeeiros de C. arabica cv. Catuaí-Vermelho, o que é comprovado pelos valores da relação Fv/Fm (Tabela 2).

A forma e disposição das folhas das duas cultivares são similares: ambas apresentam folhas opostascruzadas, curto-pecioladas, com lâminas elípticas a elíptico-lanceoladas e margens onduladas. No entanto, as folhas dos cafeeiros Conilon, no estádio de desenvolvimento estudado, são maiores, apresentando de 30 a $200 \mathrm{~mm}$ de comprimento e de 13 a $90 \mathrm{~mm}$ de largura, enquanto as de Catuaí-Vermelho apresentam comprimento entre 35 e $165 \mathrm{~mm}$ e entre 12 e $75 \mathrm{~mm}$ de largura. Isso indica que as folhas de Conilon apresentam maior área superficial para captação de luz.

Cortes transversais às lâminas foliares das duas cultivares (Figura 1) evidenciaram a maior espessura das folhas de Catuaí-Vermelho. Verificou-se que a espessura das epidermes abaxial e adaxial, e dos parênquimas paliçádico e lacunoso, contribuíram para a maior espessura total da folha (Figura 1 e Tabela 3), apesar de somente ocorrer diferença significativa entre as duas cultivares, na espessura do parênquima lacunoso.

Cortes paradérmicos às lâminas foliares revelaram que as duas cultivares diferem também quanto ao tipo e número de estômatos (Figura 2). Nos cafeeiros Catuaí-Vermelho, de 50 estômatos

Tabela 1. Fotossíntese líquida, transpiração e condutância estomática ao vapor d'água de plantas de Coffea canephora Pierre cv. Conilon e C. arabica L. cv. Catuaí-Vermelho(1).

\begin{tabular}{lccc}
\hline Cultivar & $\begin{array}{c}\text { Fotossíntese líquida } \\
\left(\mu \mathrm{mol} \mathrm{m} \mathrm{s}^{-1}\right)\end{array}$ & $\begin{array}{c}\text { Transpiração } \\
\left(\mathrm{mmol} \mathrm{m}^{-2} \mathrm{~s}^{-1}\right)\end{array}$ & $\begin{array}{c}\text { Condutância estomática } \\
\left(\mathrm{mol} \mathrm{m}^{-2} \mathrm{~s}^{-1}\right)\end{array}$ \\
\hline Conilon & $4,30 \mathrm{a}$ & $2,81 \mathrm{a}$ & $0,137 \mathrm{a}$ \\
Catuaí-Vermelho & $2,32 \mathrm{~b}$ & $1,39 \mathrm{~b}$ & $0,091 \mathrm{~b}$ \\
\hline
\end{tabular}

${ }^{(1)}$ Médias seguidas pela mesma letra, nas colunas, não diferem entre si a 5\% de probabilidade pelo teste de Tukey. 
Tabela 2. Emissão de fluorescência inicial, máxima (Fm) e variável $(\mathrm{Fv})$ e relação $\mathrm{Fv} / \mathrm{Fm}$ de plantas de Coffea canephora Pierre cv. Conilon e C. arabica L. cv. CatuaíVermelho( ${ }^{(1)}$.

\begin{tabular}{lcccc}
\hline Cultivar & \multicolumn{3}{c}{ Emissão de fluorescência } \\
\cline { 2 - 5 } & Inicial & Máxima & Variável & $\begin{array}{c}\text { Relação } \\
\text { Fv/Fm }\end{array}$ \\
\hline Conilon & $711,60 \mathrm{~b}$ & $2852,0 \mathrm{~b}$ & $2098,4 \mathrm{a}$ & $0,736 \mathrm{a}$ \\
Catuaí-Vermelho & $862,00 \mathrm{a}$ & $2959,4 \mathrm{a}$ & $2120,0 \mathrm{a}$ & $0,716 \mathrm{~b}$ \\
\hline
\end{tabular}

${ }^{(1)}$ Médias seguidas pela mesma letra, nas colunas, não diferem entre si a 5\% de probabilidade pelo teste de Tukey.

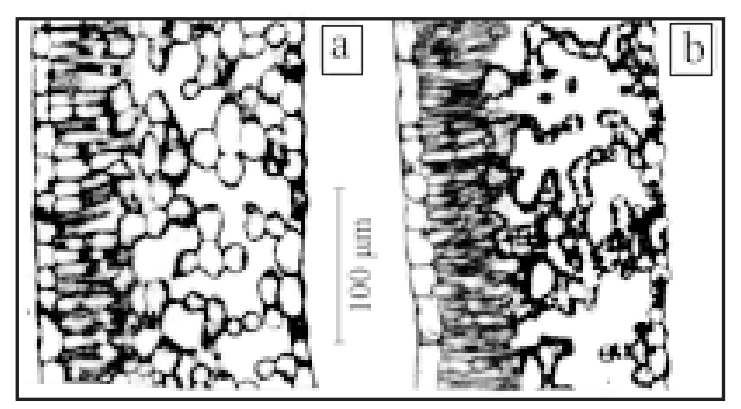

Figura 1. Fotomicrografias de lâminas foliares, em cortes transversais, de C. arabica L. cv. Catuaí-Vermelho (a) e C. canephora Pierre cv. Conilon (b).

Tabela 3. Espessura total da lâmina foliar, espessura das epidermes (abaxial + adaxial), dos parênquimas paliçádico e lacunoso de plantas de Coffea canephora cv. Conilon e C. arabica cv. Catuaí-Vermelho ${ }^{(1)}$.

\begin{tabular}{|c|c|c|c|c|}
\hline \multirow[t]{3}{*}{ Cultivar } & \multicolumn{4}{|c|}{ Espessura da lâmina foliar $(\mu \mathrm{m})$} \\
\hline & \multirow[t]{2}{*}{ Epidermes } & \multicolumn{2}{|c|}{ Parênquima } & \multirow[t]{2}{*}{ Total } \\
\hline & & Paliçádico & Lacunoso & \\
\hline Conilon & $3,20 \mathrm{a}$ & $5,50 \mathrm{a}$ & $12,80 \mathrm{~b}$ & $21,50 \mathrm{~b}$ \\
\hline Catuaí-Vermelho & $3,54 \mathrm{a}$ & $6,36 \mathrm{a}$ & $13,40 \mathrm{a}$ & $23,30 \mathrm{a}$ \\
\hline
\end{tabular}

${ }^{(1)}$ Médias seguidas pela mesma letra, nas colunas, não diferem entre si a 5\% de probabilidade, pelo teste de Tukey. observados, 35 são paracíticos, enquanto no cafeeiro Conilon há uma predominância do tipo actinocítico. As plantas de Conilon, além disso, apresentam maiores valores de índice estomático, e estômatos menores (Figura 2 e Tabela 4). Essas ca-
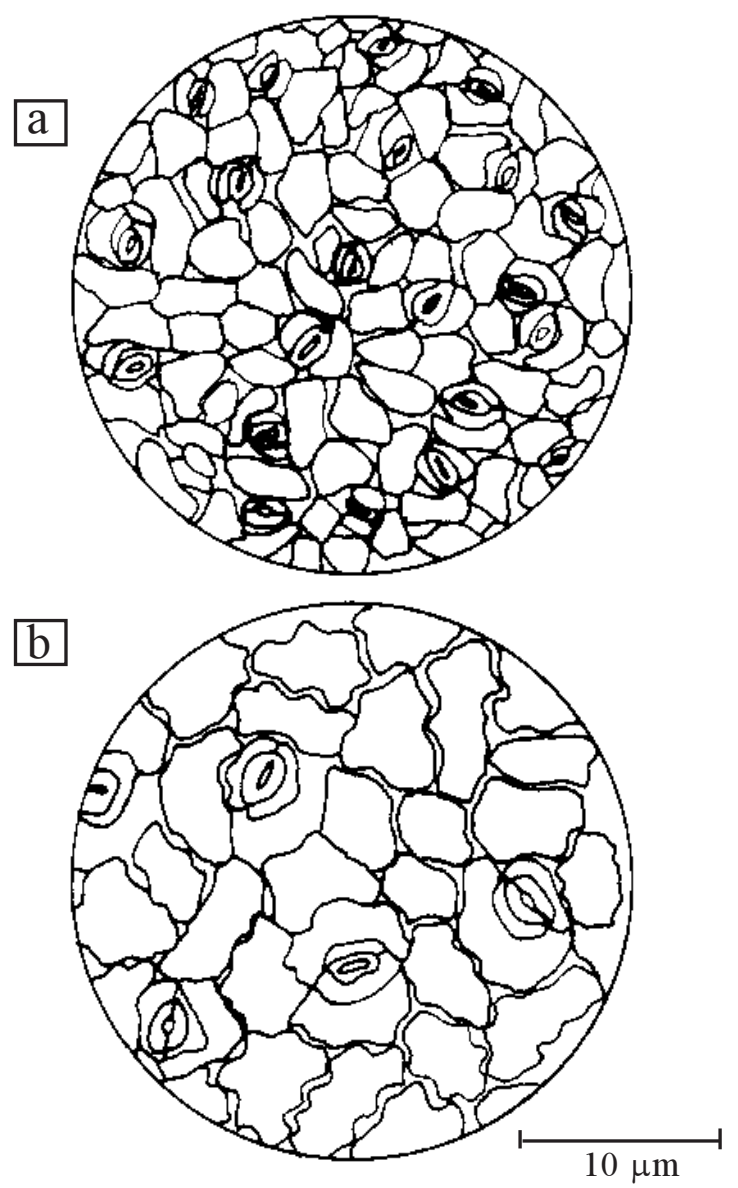

Figura 2. Representação dos estômatos de cafeeiros Coffea canephora Pierre cv. Conilon (a) e C. arabica L. cv. Catuaí-Vermelho (b), obtidos por meio de cortes paradérmicos às lâminas foliares.

Tabela 4. Comprimento e largura das células epidérmicas propriamente ditas e dos estômatos (ostíolo + células-guardas) e índice estomático em folhas de Coffea canephora cv. Conilon e C. arabica cv. Catuaí-Vermelho.

\begin{tabular}{|c|c|c|c|c|c|}
\hline \multirow[t]{2}{*}{ Cultivar } & \multicolumn{2}{|c|}{ Célula epidérmica } & \multicolumn{3}{|c|}{ Estômato } \\
\hline & Comprimento & Largura & Comprimento & Largura & Índice estomático \\
\hline & 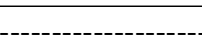 & ----- & ------------- & - & $(\%)$ \\
\hline Conilon & 57 & 47 & 51 & 52 & 25 \\
\hline Catuaí-Vermelho & 108 & 66 & 60 & 50 & 16 \\
\hline
\end{tabular}


racterísticas são semelhantes às descritas por Sondahl et al. (1976) em cafeeiros de C. canephora Pierre cv. Guarini.

Apesar de o cafeeiro Catuaí-Vermelho apresentar camadas maiores de tecidos fotossintetizantes (Tabela 3), as plantas de Conilon apresentam maior área superficial para troca de gases, maior número de estômatos na epiderme foliar abaxial (Tabela 4 e Figura 2) e maior eficiência do sistema antena (Tabela 2). Essas características ajudam a explicar as maiores taxas de condutância estomática, transpiratória e fotossintética das plantas de Conilon.

\section{Conclusões}

1. As duas cultivares de cafeeiro, Coffea arabica L. cv. Catuaí-Vermelho e C. canephora Pierre cv. Conilon, diferem quanto aos valores das taxas de fotossíntese líquida, de transpiração e de condutância estomática, sendo a segunda significativamente superior quanto a esses parâmetros.

2. Quanto à espessura foliar total, espessura da epiderme e dos parênquimas paliçádico e lacunoso, o cafeeiro da espécie $C$. arabica cv. Catuaí-Vermelho apresenta os maiores valores.

3. As duas cultivares de cafeeiro diferem morfologicamente quanto às dimensões das células epidérmicas, sobretudo quanto às dimensões dos estômatos, que são maiores nos cafeeiros de C. arabica cv. Catuaí-Vermelho.

4. Os cafeeiros das espécies Coffea arabica cv. Catuaí-Vermelho e C. canephora cv. Conilon diferem quanto ao tipo e número de estômatos predominante na epiderme foliar: actinocíticos, em maior número em Conilon, e paracíticos em CatuaíVermelho.

\section{Referências}

BERLYN, G. P.; MIKSCHE, J. P. Botanical microtechnique and cytochemistry. Ames : Iowa State University Press, 1976. 326 p.

BIERHUIZEN, J. F.; NUNES, M. A.; PLOEGMAN, C. Studies on the productivity of coffee. II. Effect of soil moisture on photosynthesis and transpiration of Coffea arabica. Acta Botanica Neerlandica, Amsterdam, v. 18 p. $367-374,1969$.
BORIN, J. Introdução ao estudo do café. São Paulo : Guarany, 1971. $144 \mathrm{p}$.

CARELLI, M. L. C.; FAHL, J. I.; TRIVELIN, P. C. O.; VOLTAN, R. B. Q. Carbon isotope discrimination and gas exchange in Coffea species grown under different irradiance regimes. Revista Brasileira de Fisiologia Vegetal, Londrina, v. 11, p. 63-68, 1999.

DA MATTA, F. M. Desempenho fotossintético do cafeeiro em resposta a tensões abióticas. Viçosa : UFV, 1995. 67 p. Tese de Doutorado.

DEDECCA, D. M. Anatomia e desenvolvimento ontogenético de Coffea arabica $\mathrm{L}$. var. typica Cramer. Bragantia, Campinas, v. 16, p. 315-366, 1957.

FAZUOLI, L. C. Genética e melhoramento do cafeeiro. In: RENA, A. B.; MALAVOLTA, E.; ROCHA, M.; YAMADA, T. (Ed.). Cultura do cafeeiro: fatores que afetam a produtividade. Piracicaba : Potafos, 1986. p. 87-106.

FRISCHKNECHT, P. M.; ELLER, B. M.; BAUMANN, T. W. Purine alkaloid formation and $\mathrm{CO}_{2}$ gas exchange in dependence of development and of environmental factors in leaves of Coffea arabica L. Planta, Berlin, v. 156, p. 295-301, 1982 .

KUMAR, D.; TIESZEN, L. L. Photosynthesis in Coffea arabica. I. Effects of light and temperature. Experimental Agriculture, Cambridge, Grã-Bretanha, v. 16, p. $13-19,1980$

NUNES, M. A.; BIERHUIZEN, J. F.; PLOEMAN, C. Studies on productivity of coffee. I. Effect of light, temperature and $\mathrm{CO}_{2}$ concentration on photosynthesis of Coffea arabica. Acta Botanica Neerlandica, Amsterdam, v. 17, p. 93-102, 1968.

NUNES, M. A.; BIERHUIZEN, J. F.; PLOEMAN, C. Studies on productivity of coffee. III. Differences in photosynthesis between four varieties of coffee. Acta Botanica Neerlandica, Amsterdam, v. 18, p. 420-424, 1969.

NUTMAN, F. J. Studies of the physiology of Coffea arabica. I. Photosynthesis of coffee leaves under natural conditions. Annals of Botany, London, v. 1, p. 353-367, 1937.

SONDAHL, M. R. I.; CROCOMO, O. J. Medida da eficiência fotossintética em cafeeiros utilizando ${ }^{14} \mathrm{CO}_{2}$. Arquivos de Biologia e Tecnologia, Curitiba, v. 17, p. 11-23, 1974.

SONDAHL, M. R. I.; CROCOMO, O. J.; SODEK, L. Measurement of ${ }^{14} \mathrm{C}$ incorporation by illuminated intact 
leaves of coffee plants from gas mixtures containing ${ }^{14} \mathrm{CO}_{2}$ Journal of Experimental Botany, Oxford, v. 27, p. $1187-1195,1976$

TIÓ, M. A. Effect of light intensity on the rate of apparent photosynthesis in coffee leaves. University of Puerto Rico Journal of Agriculture, Rio Piedras, v. 46, p. 159-166, 1962.
VOLTAN, R. B. Q.; FAHL, J. I.; CARELLI, M. L. C. Variação na anatomia foliar de cafeeiros submetidos a diferentes intensidades luminosas. Revista Brasileira de Fisiologia Vegetal, Londrina, v. 4, p. 99-105, 1992.

YAMAGUCHI, T.; FRIEND, D. J. C. Effect of leaf age and irradiance on photosynthesis of Coffea arabica. Photosynthetica, Dordrecht, v. 13, p. 271-278, 1979. 